\title{
NGAL and cystatin C: two possible early markers of diabetic nephropathy in young patients with type 1 diabetes mellitus: one year follow up
}

\author{
Nektraria Papadopoulou-Marketou, ${ }^{1,2}$ Chrysanthi Skevaki, ${ }^{3}$ Ioanna Kosteria, ${ }^{1}$ \\ Melpomeni Peppa, ${ }^{4}$ George P. Chrousos, ${ }^{1,5}$ Ioannis Papassotiriou, ${ }^{3}$ \\ Christina Kanaka-Gantenbein ${ }^{1}$
}

${ }^{1}$ Diabetes Centre of the Division of Endocrinology, Diabetes and Metabolism of the First Department of Pediatrics University of Athens, Aghia Sophia Children's Hospital, Athens, Greece; ${ }^{2}$ Section of Endocrinology, Department of Medical and Health Sciences, Linköping University, Linköping, Sweden; ${ }^{3}$ Department of Clinical Biochemistry, Aghia Sophia Children's Hospital, ${ }^{4}$ Division of Endocrinology, "Attikon" Hospital; Athens, Greece; ${ }^{5}$ King Abdulaziz University, Jeddah, S. Arabia

\begin{abstract}
OBJECTIVE: Diabetic nephropathy constitutes a major long-term complication in patients with type 1 diabetes mellitus (T1D) and its diagnosis is based on microalbuminuria. The aim of this observational follow-up study was to explore the role of neutrophil-gelatinase-associated lipocalin (NGAL) and cystatin C in unravelling early diabetic nephropathy even in patients with normoalbuminuria. DESIGN: Fifty-six euthyroid patients with T1D, with mean age 13.1 (SD: 3.2) years, and 49 healthy controls with mean age 12.8 (SD: 6.6) were recruited. Besides standard blood chemistry and urinary albumin excretion, serum NGAL (ELISA) and cystatin C (nephelometry) were measured at enrollment and after 12-15 months. GFR was calculated with the bedside Schwartz formula (eGFR) and the Lund strategy formula (L-eGFR). RESULTS: At baseline, mean NGAL levels were not significantly different between children with diabetes and controls. At re-evaluation, mean NGAL value and mean eGFR value in patients with diabetes were increased $(p=0.032$ and $p=0.003$ respectively). At both baseline and reevaluation, NGAL was positively correlated with cystatin $\mathrm{C}(\mathrm{r}=0.41, \mathrm{p}<0.001)$, systolic arterial pressure $\mathrm{z}$-score $(r=0.3, p=0.031)$ and creatinine $(r=0.32, p=0.010)$. NGAL correlated negatively with eGFR $(r=-0.26, p=0.049)$ and $L-e G F R(r=-0.33, p=0.010)$. Cystatin $C$ had a negative correlation to eGFR $(r=-0.29, p=0.025)$ and a positive one with creatinine $(r=0.35, p=0.009)$ at reevaluation. No statistically significant correlation was found between cystatin $\mathrm{C}$ and microalbuminuria $(p=0.736)$. CONCLUSIONS: NGAL and cystatin $C$, known markers of renal injury, correlate with renal function decline in T1D, suggesting that they may be used as supplementary tests to urine albumin excretion in order to unmask early renal dysfunction.
\end{abstract}

Key words: Cystatin C, diabetes-induced nephropathy, NGAL, type 1 diabetes, youth 


\section{INTRODUCTION}

Diabetes mellitus type 1 (T1D) is an infrequent but serious autoimmune disease in childhood. Despite advances in T1D treatment, diabetic complications are still a major concern since they constitute the main cause of morbidity and mortality in these patients. The most devastating complication is diabetic nephropathy (DN), which is associated with a markedly increased risk of end-stage renal failure, cardiovascular disease and premature death. It has been previously reported that childhood onset T1D is associated with a four-fold increase in the overall standardized mortality rate. ${ }^{1}$

In both the USA and Europe, around 20\% of T1D patients develop DN and progress to end-stage renal disease (ESRD). Since age at onset of T1D is younger than that of type 2 diabetes mellitus (T2D), ESRD most often develops at an earlier age in T1D, during the period of most productive years. This represents a major burden to the patients themselves and the society they live in., ${ }^{2,3}$

Nowadays, the screening of DN is mainly based on microalbuminuria (MA) assessment ${ }^{4}$ and MA may be found in $12-16 \%$ of adolescents with T1D. ${ }^{5}$ In early stages, regression to normoalbuminuria is frequently observed. ${ }^{6}$ Puberty itself and poor glycemic control are independent risk factors for MA in patients with T1D. ${ }^{7}$ However the diagnostic role of microalbuminuria in DN has recently been disputed by several authors, while it has been widely proposed that other biomarkers are needed for early identification of renal lesions. ${ }^{2,8-10}$ Previous studies have shown evidence of several clinical and laboratory predictors for DN, such as increased systolic blood pressure, even within the pre-hypertensive range ${ }^{11}$ and hyperlipidemia identifying subjects at risk for early generalized endotheliopathy and cardiovascular disease (CVD) as well. ${ }^{12-14}$ Among T1D patients with nephropathy and hypertension, $50 \%$ will go on to develop end-stage renal disease within a decade. ${ }^{4}$

The pathophysiologic changes in DN that lead to renal function decline are associated with cellular and extracellular derangements in both the glomerular and tubulo-interstitial compartments. ${ }^{12,13}$ Several studies have reported that normoalbuminuric subjects, including prepubertal children with longstanding diabetes, often have glomerular basement membrane (GBM) thickening, mesangial expansion ${ }^{15-17}$ and significant glomerulopathy lesions. ${ }^{18}$

Glomerular and renal tubular interstitium injury plays a role in the pathogenesis of $\mathrm{DN}^{19}$ and several tubular damage markers have been investigated in the early detection of DN. ${ }^{9,19,20}$ Among them, neutrophil gelatinase-associated lipocalin (NGAL), which is a 178 amino acid $25 \mathrm{kDa}$ protein, first purified and identified in 1993 by Kjeldsen et al, seems to be a promising biomarker. ${ }^{9,19,21}$ It is mainly produced in renal tubules in response to structural kidney injury, ${ }^{22}$ but also, to a lesser degree, in the lung, trachea, stomach, and colon, while it is excreted in the urine. ${ }^{23}$ NGAL values may be influenced by renal diseases, hypertension, inflammatory conditions, hypoxia and malignancies. ${ }^{22}$ NGAL as a renal biomarker was first described in 2003, following experimental renal ischemia in a mouse model. ${ }^{24}$ In contrast to conventional serum markers, such as creatinine or blood urea nitrogen, NGAL is considered as a marker of renal structural damage. In previous studies, NGAL was effective in the early diagnosis of acute kidney injury (AKI) in several clinical settings ${ }^{25}$ and was also validated for its prognostic role in cardiovascular morbidity. ${ }^{26,27}$

Cystatin C (CysC) is a small-molecular-weight protein and, in particular, an endogenous cysteine proteinase inhibitor highly correlated with GFR. ${ }^{28}$ It is not influenced by inflammatory conditions, muscle mass, gender, body composition and age (after the age of 12 months) ${ }^{29}$ Superiority of CysC over other markers of renal function decline lies in its ability to remain unbound to protein and to be freely filtered across the glomeruli. In healthy subjects, CysC is almost freely filtered by the glomeruli and almost entirely reabsorbed in the proximal tubule like other low molecular weight proteins with no or only partial tubular secretion. Inter-individual variation in CysC account for $25 \%$ of its biological variability compared to $93 \%$ for creatinine. ${ }^{29,30}$ Moreover, increased CysC values are associated with increased cardiovascular morbidity risk and atherosclerosis progression in both T1D patients and severely obese children..$^{31,32}$

Glomerular filtration rate (GFR) is best evaluated by the clearance of iohexol (iGFR). ${ }^{32,33}$ Alternatively, in children, the 2009 modified Schwartz bedside GFR 
formula has been widely adopted because it has been strongly correlated to iGFR. ${ }^{34,35}$ Moreover, formulas that assess both creatinine and cystatin $\mathrm{C}$, like the Lund strategy (L-eGFR), ${ }^{36}$ are considered to provide more accurate and reliable estimation of the GFR.

The aim of this study was to determine the possible predicting roles of serum NGAL and serum $\mathrm{CysC}$, as supplementary tests to the urinary albumin excretion, in unmasking an early renal structural injury and renal function decline in asymptomatic, normoalbuminuric young T1D patients.

\section{RESEARCH DESIGN AND METHODS}

This is an observational cross-sectional long-term follow-up study. During a 12-15 months' follow-up period, a patient-group that completed both baseline and reevaluation at 12-15 months was evaluated. The patient group consisted of 56 T1D patients, with mean age 13.1 years (SD: 3.20$)$ and mean diabetes duration 4.59 years (SD: 3.49 ) at enrollment, who were prospectively followed for at least 2 years at the Diabetes Centre of the First Department of Pediatrics of the University of Athens, Aghia Sophia Children's Hospital, Greece. The follow-up is scheduled to continue and reevaluation of the biomarkers will take place at a third point of time, three years after the baseline assessment.

The diagnosis of T1D was based in all participants of the patients' group on the presence of the high titer of at least one and mostly two of the known autoantibodies related to type 1 diabetes mellitus. Specifically, the percentage (\%) of positivity for each autoantibody tested was: for Glutamic Acid Decarboxylase Autoantibodies (GADA) (72\%), Insulinoma-Associated-2 Autoantibodies (IA2) (71\%), Insulin Autoantibodies (IAA) (45\%) and Islet Cell Cytoplasmic Autoantibodies (ICA) (35\%). Ten of the patients presented with microalbuminuria at inclusion in the study, while five of them had persistent microalbuminuria at reevaluation and five patients were restored to normoalbuminuria. Three patients presented with newly diagnosed microalbuminuria at reevaluation. In total, eight patients were found to have microalbuminuria at reassessment.

Forty-nine healthy children with mean age 12.8 (SD: 6,6) who were referred to the Division of Endocrinology for growth evaluation but were found to be within the normal reference charts and willing to participate in the study, served as controls (Table 1). Informed consent was obtained from the parents of

Table 1. Demographic characteristics of T1D patients at baseline and conrols

\begin{tabular}{|c|c|c|c|c|c|c|c|c|}
\hline & \multicolumn{4}{|l|}{ T1D } & \multicolumn{3}{|l|}{ Contrals } & P-value \\
\hline $\mathrm{n}$ & \multicolumn{4}{|l|}{56} & \multicolumn{3}{|l|}{49} & \\
\hline \multirow[t]{3}{*}{ Gender } & \multirow{2}{*}{\multicolumn{4}{|c|}{$\begin{array}{l}\text { Aars } 57.1 \%(n=32) \\
\text { Girls } 42.9 \%(n=24)\end{array}$}} & \multirow{2}{*}{\multicolumn{3}{|c|}{$\begin{array}{l}\text { Boys } 42.8 \%(n=21) \\
\text { Girls } 57.2 \%(n=28)\end{array}$}} & 0.31 \\
\hline & & & & & & & & \\
\hline & Mean & $95 \% \mathrm{Cl}$ & \multicolumn{2}{|l|}{ SD } & Mean & $95 \% \mathrm{Cl}$ & SD & \\
\hline Age (years) & 13.1 & $12.2-13.9$ & \multicolumn{2}{|l|}{3.2} & 12.8 & $10.9 \cdot 14.7$ & 6.6 & 0.21 \\
\hline Age of diagnosis (years) & 8.5 & $7.6-9.5$ & \multicolumn{2}{|l|}{3.4} & \multicolumn{4}{|l|}{ na } \\
\hline BMII z-score & 0.33 & $0.04-0.62$ & 0.8 & & 0.32 & $0.02-0.61$ & 0.8 & 0.68 \\
\hline HbA1e & $\begin{array}{l}8.36 \%(68 \\
\mathrm{mmal} / \mathrm{mol})\end{array}$ & $\begin{array}{l}7.88-8.85 \%(63- \\
\mathrm{mmal} / \mathrm{mol})\end{array}$ & 73 & 1.7 & $\begin{array}{l}4.70 \%(28 \\
\mathrm{mmol} / \mathrm{mal})\end{array}$ & $\begin{array}{l}4.4-4.9 \%(25-30 \\
\mathrm{mmal} / \mathrm{mol})\end{array}$ & 0.4 & $<0.001$ \\
\hline T1D duration (years) & 4.5 & $3.6-5.5$ & & 3.4 & na & & & \\
\hline $\begin{array}{l}\text { Microslbuminuria } \\
\text { (daily secretion of urinary albumin } \\
230 \mathrm{mg} \text { but } 5300 \mathrm{mg} \text { ) }\end{array}$ & \multicolumn{4}{|c|}{$18.5 \%$ presented micraalbuminuria $(10 / 56)$} & \multicolumn{3}{|c|}{$0 \%$ presented micraalbuminuria $(0 / 49)$} & \\
\hline
\end{tabular}

na: not applicable. 
all participants prior to their inclusion in the study.

The study was approved by the Ethics Committee of the Aghia Sophia Children's Hospital and has therefore been performed in accordance with the ethical standards laid down in the 1964 Declaration of Helsinki and its later amendments.

Inclusion criteria for all participants were euthyroidism for at least 6 months prior to study enrollment as well as during follow-up. The exclusion criteria were the presence of active urinary tract infection, glucocorticoid medication, pregnancy, renal disease and any chronic disease other than T1D.

After 12-hour fasting, a morning blood sample was obtained for blood urea nitrogen, creatinine ( $\mathrm{sCr}$ ), uric acid (UA) and Glycated hemoglobin (HbA1c) determination. Blood was collected in special vials to be used for measurement of the specific markers NGAL and CysC. Part of the blood was centrifuged for separation of serum and stored at $-80^{\circ} \mathrm{C}$, until final assessments. Urinary albumin excretion was determined from a 24-hour urine collection (microalbuminuria was defined with values between 30$300 \mathrm{mg} / 24$ hours, measured on at least two of three measurements over a two- to three-month period) and was measured by nephelometry.

CysC concentration was measured by an immuno-nephelometric technique using the BN Prospec nephelometer (Dade Behring, Siemens Healthcare Diagnostics, Liederbach, Germany). The nephelometric method is more sensitive and performs optimally in dilute solution, making it preferable for small sample volumes encountered in the pediatric population. With a range of $0.23-7.25 \mathrm{mg} / \mathrm{L}$, this assay is currently the most precise automated assay across the clinical concentration range. The inter-assay coefficient of variation (CV) for the assay was $5.05 \%$ and $4.87 \%$ at mean concentrations of 0.97 and 1.90 $\mathrm{mg} / \mathrm{L}$, respectively.

The GFR was calculated according to the eGFR ${ }^{35}$ and L-eGFR ${ }^{36}$ equation methods mentioned above.

Serum NGAL levels were measured using a commercially available ELISA (Bioporto, Gentofte, Denmark). The intra- and inter-assay coefficients of variation $(\mathrm{CVs})$ were $5.6 \%$ and $6.4 \%$, respectively.

The values of systolic and diastolic arterial blood pressure (SAP and DAP respectively), body mass index (BMI) and the height are expressed in z-scores.

\section{Statistical analysis}

Statistical analyses were performed using MedCalc for Windows, version 12.5 (MedCalc Software, Ostend, Belgium). Correlation analysis is used to determine whether the values of two variables are associated using Pearson parametric correlation (Pearson's correlation coefficient $r$ with P-value). Student t-test was performed as appropriate (paired samples t-test were used to test the null hypothesis that the average of the differences between a series of paired observations is zero when performed on the same subjects or independent samples t-test when performed between controls' and patients' group). Multiple regression analysis was a method used to examine the relationship between one dependent variable and one or more independent variables. The significance was defined at $\mathrm{p}$-value $<0.05$, rho and $95 \%$ confidence interval (CI) for the correlation coefficient.

\section{RESULTS}

Paired samples t-test of repeated measurements revealed that mean eGFR value in T1D patients was increased at re-evaluation (mean eGFR at baseline $=$ 90.72 with $\mathrm{SD}=19.8$, mean eGFR at reevaluation $=$ 97.5 with $\mathrm{SD}=17.5, \mathrm{n}=56, \mathrm{p}=0.003)$. The same analysis showed an increase of the L-eGFR at reevaluation, but this result was not statistically significant (mean value at baseline $=120.7$, mean value at reevaluation= 122.7, $\mathrm{n}=56$ ). Regarding eGFR, 13 patients were found to have a decreased value at reevaluation, while for L-eGFR, 23 patients had a decreased value at reevaluation. Mean value of NGAL at baseline was not significantly different between T1D patients and controls (mean: 59.5 for T1D patients, mean for controls: $62.6, \mathrm{p}=0.393$ ), but NGAL mean value of T1D patients at reevaluation (mean: 67.6) was statistically significantly higher than the mean value of the controls' group $(\mathrm{p}<0.001)$ according to Student t-test statistical analysis for independent samples. Paired samples t-test of repeated measurements revealed that mean NGAL value in T1D patients was increased at re-evaluation $(\mathrm{p}=0.032)$ (Figure 1). At baseline, regarding the T1D patients' group, NGAL had no 


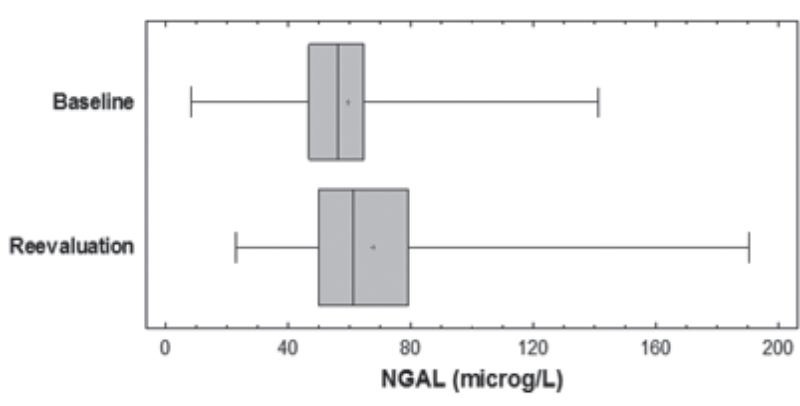

Figure 1. Comparison of NGAL plasma lenels at baseline and at re-evaluation after one year $(p=0.032)$. Boxes represent the interquartile range; lines inside boxes represent the median value; cross represents mean marker; whiskers represent the lowest and highest observation, respectively.

significant correlation either with eGFR $(p=0.067)$ or with CysC $(\mathrm{p}=0.179)$, but it was negatively correlated with L-eGFR $(\mathrm{r}=-0.35, \mathrm{p}=0.007, \mathrm{n}=56)$. At re-evaluation, NGAL was positively correlated to CysC $(r=0.41, p=0.0014, n=56)$ (Figure 2), SAP zscore $(\mathrm{r}=0.29, \mathrm{p}=0.031, \mathrm{n}=56$ ) (Figure 3 ) and $\mathrm{sCr}$ $(\mathrm{r}=0.32, \mathrm{p}=0.010 \mathrm{n}=56)$. Moreover, at re-evaluation, NGAL had a negative correlation with both eGFR $(\mathrm{r}=-0.26, \mathrm{p}=0.049, \mathrm{n}=56)$ and L-eGFR $(\mathrm{r}=-0.33$, $\mathrm{p}=0.002, \mathrm{n}=56)$ (Figure 4). No statistically significant correlation was found between NGAL and microalbuminuria; however, regression analysis revealed that NGAL values higher than $70 \mu \mathrm{g} / \mathrm{L}$ at both time points of assessment had a positive correlation with the presence of microalbuminuria $(\mathrm{r}=0.29, \mathrm{p}=0.038)$.

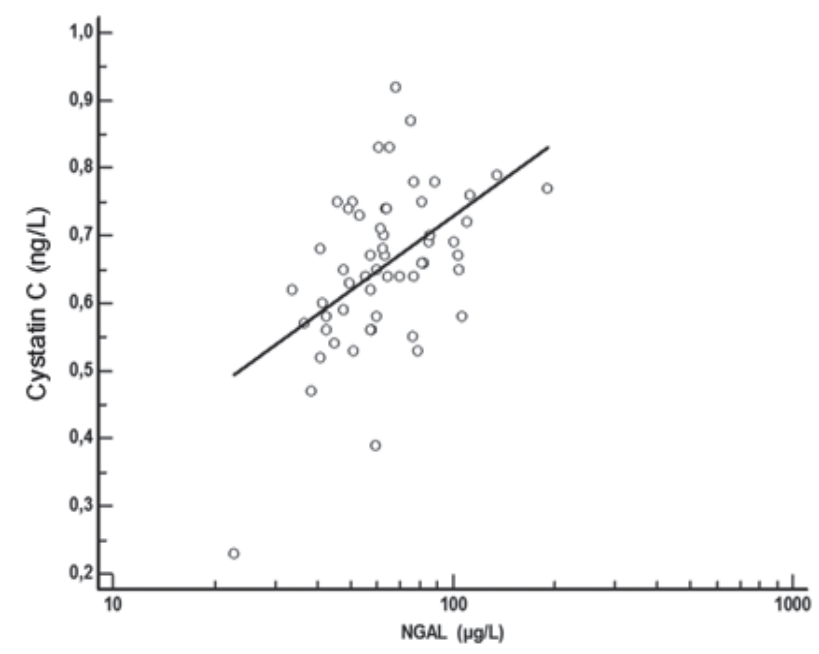

Figure 2. NGAL had a positive correlation to Cystatin $\mathrm{C}$ in patients with T1D at re-evaluation $(r=0.41, p=0.001, n=56)$.

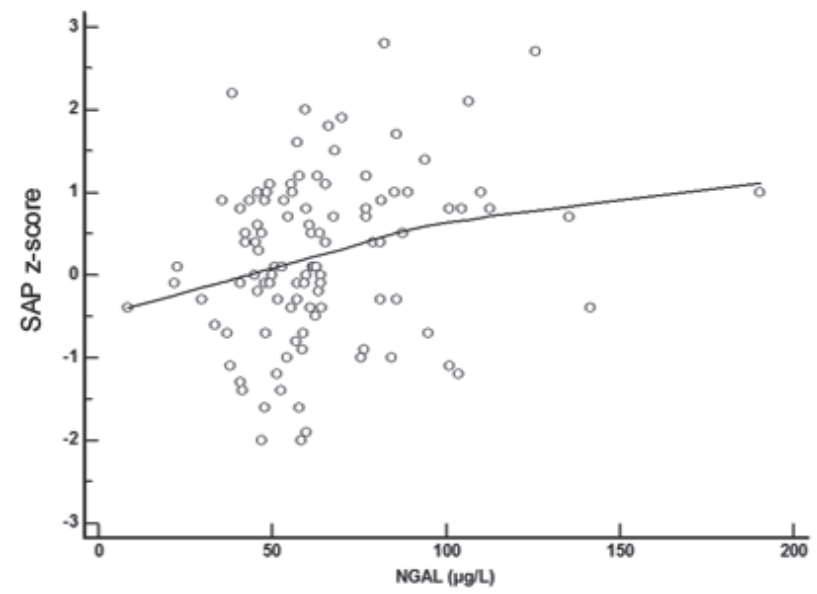

Figure 3. NGAL had a positive correlation to systolic arterial pressure (SAP) z-score in patients with type 1 diabetes mellitus at re-evaluation $(\mathrm{r}=0.3, \mathrm{p}=0.03, \mathrm{n}=56)$.

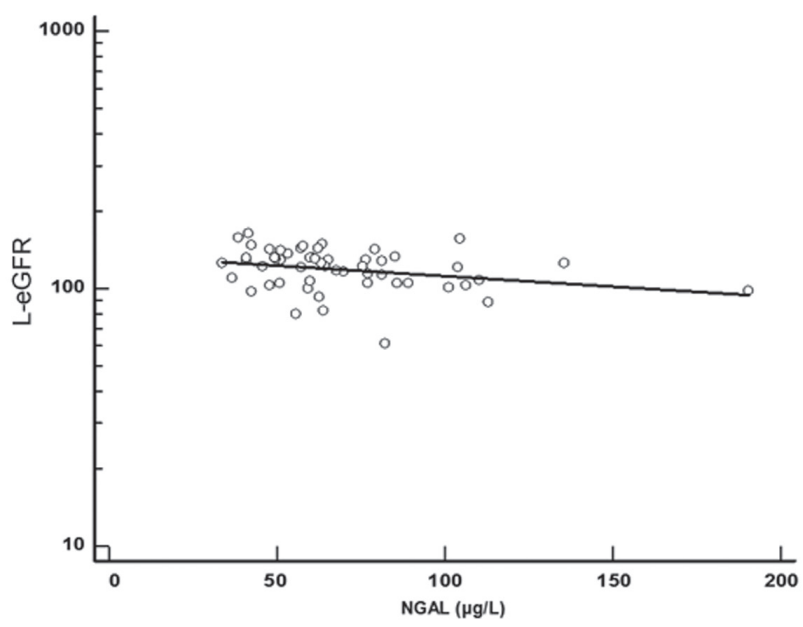

Figure 4. NGAL had a negative correlation to eGFR estimated with the Lund strategy formula in patients with type 1 diabetes mellitus at re-evaluation $(r=0.33, p=0.002, n=56)$.

NGAL value, both at baseline and at re-evaluation, did not significantly correlate either with $\mathrm{HbA} 1 \mathrm{c}$ or with BMI z-score, age or diastolic arterial pressure z-score. Furthermore, a multiple regression analysis was performed between NGAL at both time points of evaluation and age, age of T1D onset, BMI z-score, pubertal stages, sex and height z-score, but no statistically significant associations were revealed. However, higher NGAL values correlated positively with T1D duration $(\mathrm{p}=0.049)$.

Paired sample t-test of repeated measurements 
revealed that CysC mean value in T1D patients did not significantly differ between the two time points of assessment $(\mathrm{p}=0.61)$. No significant difference was observed between controls' and T1D CysC measurements $(\mathrm{p}=0.21)$. At reevaluation, $\mathrm{Cys} \mathrm{C}$ had a negative correlation to eGFR $(\mathrm{r}=-0.29, \mathrm{p}=0.025, \mathrm{n}=56)$ and a positive one with $\mathrm{sCr}(\mathrm{r}=0.35, \mathrm{p}=0.009, \mathrm{n}=56)$. At re-evaluation, UA in T1D was positively correlated with CysC $(r=0.41, p=0.016, n=56)$. No statistically significant correlation was found between CysC and microalbuminuria $(p=0.75), \mathrm{HbA1c}(\mathrm{p}=0.24), \mathrm{BMI}$ $z$-score $(p=0.38)$, age $(p=0.79)$, SAP $z$-score $(p=0.38)$, DAP $z$-score $(p=0.46)$ and T1D duration $(p=0.44)$. A multiple regression analysis was performed between CysC at both time points of evaluation and age, age of T1D onset, T1D duration, pubertal stages, BMI z-score, sex and height z-score and a statistically significant positive correlation between CysC and male sex in the patients' group $(r=0.47, p<0.001)$ was revealed. No statistically significant correlation was found between the male sex and cystatin $\mathrm{C}$ in the controls' group. A multiple regression analysis was performed between sCreatinine at both time points of evaluation and age as well as T1D duration and a statistically significant positive correlation between age of assessment and $\mathrm{sCr}$ was found $(\mathrm{r}=0.50$, $\mathrm{p}=0.0014)$, but no significant association with T1D duration was demonstrated $(p=0.372)$. A multiple regression analysis was performed between eGFR, L-eGFR and age as well as T1D duration, but no statistically significant association was found.

No participant from the control group presented microalbuminuria.

During follow-up all participants had eGFR greater than $60 \mathrm{ml} / \mathrm{min}$.

\section{DISCUSSION}

Microalbuminuria has generally been considered as the earliest marker of diabetic nephropathy development and is often associated with established significant glomerular damage. However, recent studies showed that MA does not necessarily reflect permanent renal impairment. In addition, several lines of evidence suggest that early structural damage in both glomerular and tubular structures may be present in normoalbuminuric subjects. Indeed, cohort studies including prepubertal children with average diabetes duration of 5-8 years revealed GBM thickening and mesangial expansion, ${ }^{15}$ while it also disclosed that longstanding normoalbuminuric T1D patients may have significant glomerulopathy lesions ${ }^{16,18,19}$ The pathophysiologic changes in DN therefore include renal function decline associated with cellular and extracellular derangements in both the glomerular and tubulo-interstitial compartments. ${ }^{18}$

The first results of this long-term observational study aimed in assessing the predictive value of early markers of renal injury, such as NGAL and CysC, in young T1D patients by unraveling renal structural damage long before renal dysfunction occurs.

We found that the mean value of estimated GFR for the patients' group was increased at reevaluation, as expected, since the equation formulas take into account body height, which increases with advancing age in children and adolescents. The cases where eGFR was lower at reevaluation than at baseline were related to a higher serum creatinine or cystatin C. NGAL correlated negatively with eGFR and LeGFR, indicating an association with renal function decline. NGAL was positively correlated with CysC, SAP z-score, Cr and T1D duration. NGAL values higher than $70 \mu \mathrm{g} / \mathrm{L}$ were associated with the presence of microalbuminuria. Our study underpins the value of NGAL as a biomarker of early renal damage in T1D, since NGAL values increased during follow-up more specifically when eGFR was decreased and this may reflect a progress of the early renal structural damage occurring with advancing T1D duration. NGAL value demonstrated a significant negative correlation with the estimated GFR using two different methods, suggesting that a higher NGAL value is associated with a glomerular function decline even if remaining within normal values, this probably suggestive of the occurrence of early lesions. The number of the included patients who presented microalbuminuria was much lower than the number of patients who presented a decreased eGFR. Moreover, the fact that a percentage of normoalbuminuric patients, who were found to have renal function decline, presented higher NGAL concentrations, while some patients with microalbuminuria had normal NGAL concentrations, may imply that these two markers, i.e. microalbuminuria and NGAL, may reflect dif- 
ferent sites of renal damage during the process of DN establishment.

It is certainly necessary to identify markers of early tubular damage independently of albuminuria development in patients with early DN and progression, as it may play a significant role in the management of the normoalbuminuric renal insufficiency cases. ${ }^{36,37}$ The significant positive correlation between NGAL, a known marker of structural renal lesion, and established renal biochemical indexes such as sCr and CysC underpin its important role in the early diagnosis of renal dysfunction.

SAP has been previously demonstrated to be a predictor of DN. ${ }^{3}$ In our study, because it was specifically focused on a young population, we estimated the SAP z-score and found a positive correlation with NGAL. This fact, independently of DN, may reflect an indirect role of NGAL as an endothelial dysfunction marker. Undoubtedly, further studies investigating endothelial dysfunction will further delineate the extent of microvascular damage in DN. According to multiple regression analysis, NGAL values were not influenced by age, age of T1D onset and puberty, suggesting independency from the several physiologic mechanisms occurring during puberty.

A limitation of our study was the fact that repeated measurements were obtained only in the children with T1D after 12-15 months and not in the control group as well. However, it is considered unethical to perform repeated venipunctures in healthy children, especially in assessing markers that have been shown not to be related to pubertal progression and age, while they do not increase without a backstage tissue injury. ${ }^{23}$ Moreover, it is known that microalbuminuria does not occur in healthy children unless renal disease, hypertension, obesity or cardiovascular disease are present. $^{38}$

The diagnostic utility of CysC is well documented in the acute setting. ${ }^{28,29,39}$ Our study demonstrated that CysC had a positive correlation with $\mathrm{sCr}$ and a negative correlation with eGFR, suggesting that higher cystatin $\mathrm{C}$ values correlate with a decreased eGFR. These findings may support the predictive role of CysC in early DN. Moreover, cystatin C assessment is undoubtedly useful in the more accurate GFR determination. ${ }^{30}$ Its significant positive correlation with UA, which has been demonstrated to be associated with renal dysfunction, ${ }^{10}$ hypertension and cardiovascular disease, further supports the role of $\mathrm{Cys} \mathrm{C}$ as a prognostic cardiometabolic biomarker in diagnosing early diabetic microvascular complications. ${ }^{31,39}$ Multiple regression analyses found that CysC values were not influenced by age, duration of T1D and puberty. However, CysC was found to have higher values in boys in the patients' group, and this fact may imply a gender dimorphism of this biomarker. This finding has not been previously reported, nor has it been replicated in our control group, and it therefore necessitates further validation through large-scale future studies.

According to the National Kidney Foundation (NKF) and the Kidney Disease Outcomes Quality Initiative (KDOQI), a patient is considered to be in chronic kidney disease (CKD) state if he presents a GFR $<60$ $\mathrm{mL} / \mathrm{min}$ per $1.73 \mathrm{~m}^{2}$ for three months or more. Alternatively, any ongoing (at least three months) structural or functional abnormality of the kidney, regardless of GFR, that can be detected by pathological abnormalities or specific markers is considered CKD. ${ }^{40}$ The significant correlation between NGAL, cystatin C and GFR decline further support the prognostic role of NGAL and cystatin $\mathrm{C}$ in unmasking the structural renal damage before an overt renal impairment becomes evident. The association between the early tubular interstitial damage in normoalbuminuric people with T1D and NGAL is further supported by recently published studies. ${ }^{17,18,20}$ To our knowledge, this is the first study to demonstrate the predictive role of NGAL and CysC as early markers of DN in children, adolescents and young adults before severe overt nephropathy occurs. These findings remain to be further confirmed at the final evaluation of this long-term study as well as by further prospective studies.

Defining new predictors as supplementary tests to urinary albumin excretion for the early diagnosis of DN would accelerate the effective management and treatment approaches which are desperately needed in order to minimize the rates of severe cardiorenal morbidity and mortality in young T1D patients. Therefore, these data need to be confirmed by further large-scale longitudinal studies before being integrated in the DN risk assessment of young patients with T1D. 


\section{CONFLICT OF INTEREST}

None for all authors.

\section{FUNDING}

No conflict of interest or funding sources exist for any author.

\section{AUTHORS' CONTRIBUTIONS}

N.P. researched data and wrote the manuscript. C.S. contributed to the biochemical measurement I.K. contributed to the research of data. M.P. contributed to the research of data. G.C. contributed to the discussion and reviewed/edited the manuscript. I.P. contributed to the biochemical measurement and contributed to the discussion. C.K.-G. contributed to the discussion and reviewed/edited the manuscript.

\section{REFERENCES}

1. Nichols GA, Vupputuri S, Lau H, 2011 Medical care costs associated with progression of diabetic nephropathy. Diabetes Care 34: 2374-2378.

2. Perkins BA, Krolewski AS, 2005 Early nephropathy in type 1 diabetes: a new perspective on who will and who will not progress. Curr Diab Rep 5: 455-463.

3. Raile K, Galler A, Hofer S, et al, 2007 Diabetic nephropathy in 27,805 children, adolescents, and adults with type 1 diabetes: effect of diabetes duration, A1C, hypertension, dyslipidemia, diabetes onset, and sex. Diabetes Care 30: 2523-2528.

4. Mogensen CE, 1997 How to protect the kidney in diabetic patients: with special reference to IDDM. Diabetes 46: Suppl 2: 104-111.

5. Schultz CJ, Konopelska-Bahu T, Dalton RN, et al, 1999 Microalbuminuria prevalence varies with age, sex, and puberty in children with type 1 diabetes followed from diagnosis in a longitudinal study. Oxford Regional Prospective Study Group. Diabetes Care 22: 495-502.

6. Perkins BA, Ficociello LH, Silva KH, et al, 2003 Regression of microalbuminuria in type 1 diabetes. $\mathrm{N}$ Engl J Med 348: 2285-2293.

7. Access O, 2009 Adolescent type 1 Diabetes Cardio-renal Intervention Trial (AdDIT). BMC Pediatr 9: 79.

8. Lacquaniti A, Donato V, Pintaudi B, et al, 2013 "Normoalbuminuric" diabetic nephropathy: tubular damage and NGAL. Acta Diabetol 50: 935-942.

9. Hovind P, Rossing P, Tarnow L, et al, 2009 Serum uric acid as a predictor for development of diabetic nephropathy in type 1 diabetes: an inception cohort study. Diabetes 58: 1668-1671.
10. Perkins BA, Ficociello LH, Roshan B, et al, 2010 In patients with type 1 diabetes and new-onset microalbuminuria the development of advanced chronic kidney disease may not require progression to proteinuria. Kidney Int 77: 57-64.

11. Orchard TJ, Forrest KY, Kuller LH, Becker DJ, 2001 Lipid and blood pressure treatment goals for type 1 diabetes: 10-year incidence data from the Pittsburgh Epidemiology of Diabetes Complications Study. Diabetes Care 24: 1053-1059.

12. Caramori ML, Kim Y, Huang C, et al, 2002 Cellular basis of diabetic nephropathy: 1 . Study design and renal structural-functional relationships in patients with long-standing type 1 diabetes. Diabetes 51: 506-513.

13. Schultz CJ, Amin R, Dunger DB, 2002 Markers of microvascular complications in insulin dependent diabetes. Arch Dis Child 87: 10-12.

14. Orchard TJ, 1994 From diagnosis and classification to complications and therapy. DCCT. Part II? Diabetes Control and Complications Trial. Diabetes Care 17: 326-338.

15. Ellis EN, Warady BA, Wood EG, et al, 1997 Renal structural-functional relationships in early diabetes mellitus. Pediatr Nephrol 11: 584-591.

16. Drummond KN, Kramer MS, Suissa S, et al, 2003 Effects of duration and age at onset of type 1 diabetes on preclinical manifestations of nephropathy. Diabetes 52: 1818-1824.

17. Steinke JM, Sinaiko AR, Kramer MS, et al, 2005 The early natural history of nephropathy in type 1 diabetes. Diabetes 54: 2164-2171.

18. Torbjörnsdotter TB, Perrin NESS, Jaremko GA, Berg UB, 2005 Widening of foot processes in normoalbuminuric adolescents with type 1 diabetes. Pediatr Nephrol 20: 750-758.

19. Nauta FL, Boertien WE, Bakker SJL, et al, 2011 Glomerular and tubular damage markers are elevated in patients with diabetes. Diabetes Care 34: 975-981.

20. Chae H-W, Shin J-I, Kwon A-R, et al, 2012 Spot urine albumin to creatinine ratio and serum cystatin $\mathrm{C}$ are effective for detection of diabetic nephropathy in childhood diabetic patients. J Korean Med Sci 27: 784-787.

21. Thrailkill KM, Moreau CS, Cockrell GE, et al, 2010 Disease and gender-specific dysregulation of NGAL and MMP-9 in type 1 diabetes mellitus. Endocrine 37: 336-343.

22. Schmidt-Ott KM, Mori K, Li JY, et al, 2007 Dual action of neutrophil gelatinase-associated lipocalin. J Am Soc Nephrol 18: 407-413.

23. Devarajan P, 2010 Neutrophil gelatinase-associated lipocalin: a promising biomarker for human acute kidney injury. Biomark Med 4: 265-280.

24. Bagshaw SM, Bennett M, Haase M, et al., 2010 Plasma and urine neutrophil gelatinase-associated lipocalin in septic versus non-septic acute kidney injury in critical illness. Intensive Care Med 36: 452-461. 
25. Haase-Fielitz A, Haase M, Devarajan P, 2014 Neutrophil gelatinase-associated lipocalin as a biomarker of acute kidney injury: a critical evaluation of current status. Ann Clin Biochem 51: 335-351.

26. McKittrick IB, Bogaert Y, Nadeau K, et al, 2011 Urinary matrix metalloproteinase activities: biomarkers for plaque angiogenesis and nephropathy in diabetes. Am J Physiol Renal Physiol 301: 1326-1333.

27. Hawkins R, 2011 New biomarkers of acute kidney injury and the cardio-renal syndrome. Korean J Lab Med 31: 72-80.

28. Dharnidharka VR, Kwon C, Stevens G, 2002 Serum cystatin $\mathrm{C}$ is superior to serum creatinine as a marker of kidney function: a meta-analysis. Am J Kidney Dis 40: 221-226.

29. Finney H, Newman DJ, Thakkar H, et al, 2000 Reference ranges for plasma cystatin $\mathrm{C}$ and creatinine measurements in premature infants, neonates, and older children. Arch Dis Child 82: 71-75.

30. Grubb A, Björk J, Nyman U, et al, 2011 Cystatin C, a marker for successful aging and glomerular filtration rate, is not influenced by inflammation. Scand J Clin Lab Invest 71: 145-149.

31. Maahs DM, Ogden LG, Kretowski A, et al, 2007 Serum Cystatin C Predicts Progression of Subclinical Coronary Atherosclerosis in Individuals With Type 1 Diabetes. Diabetes 56: 2774-2779.

32. Codoñer-Franch P, Ballester-Asensio E, Martínez-Pons L, et al, 2011 Cystatin C, cardiometabolic risk, and body composition in severely obese children. Pediatr
Nephrol 26: 301-307.

33. Brown SC, O’Reilly PH, 1991 Iohexol clearance for the determination of glomerular filtration rate in clinical practice: evidence for a new gold standard. J Urol 146: 675-679.

34. Schwartz GJ, Schneider MF, Maier PS, et al, 2012 Improved equations estimating GFR in children with chronic kidney disease using an immunonephelometric determination of cystatin C. Kidney Int 82: 445-453.

35. Schwartz GJ, Work DF, 2009 Measurement and estimation of GFR in children and adolescents. Clin J Am Soc Nephrol 4: 1832-1843.

36. Grubb A, 2010 Non-invasive estimation of glomerular filtration rate (GFR). The Lund model: Simultaneous use of cystatin C- and creatinine-based GFR-prediction equations, clinical data and an internal quality check. Scand J Clin Lab Invest 70: 65-70.

37. Perkins BA, Krolewski AS, 2009 Early nephropathy in type 1 diabetes: the importance of early renal function decline. Curr Opin Nephrol Hypertens 18: 233-240.

38. Marcun N, 2013 Microalbuminuria in pediatric patients with hypertension. Health 5: 40-46.

39. Ix JH, Shlipak MG, Chertow GM, Whooley MA, 2007 Association of cystatin C with mortality, cardiovascular events, and incident heart failure among persons with coronary heart disease: data from the Heart and Soul Study. Circulation 115: 173-179.

40. Uhlig K, Eckardt K-U, 2012 A decade after the KDOQI CKD guidelines: impact on CKD guidelines. Am J Kidney Dis 60: 705-706. 